\title{
Sintomatologia depressiva em cuidadores familiares de idosos com Alzheimer ou
}

\section{outra demência}

\author{
Depressive symptoms in family caregivers of elderly people with Alzheimer's or other dementia \\ Síntomas depresivos en cuidadores familiares de personas mayores con Alzheimer u otra demencia
}

Recebido: 15/04/2021 | Revisado: 25/04/2021 | Aceito: 29/04/2021 | Publicado: 13/05/2021

\author{
Daniela Cristina Magno \\ ORCID: https://orcid.org/0000-0003-4287-2969 \\ Universidade Federal de São Paulo, Brasil \\ E-mail: danimagnodcm@gmail.com \\ Daniel Augusto da Silva \\ ORCID: https://orcid.org/0000-0002-2716-6700 \\ Universidade Federal de São Paulo, Brasil \\ E-mail: daniel.augusto@unifesp.br \\ João Fernando Marcolan \\ ORCID: https://orcid.org/0000-0001-8881-7311 \\ Universidade Federal de São Paulo, Brasil \\ E-mail: jfmarcolan@uol.com.br
}

\begin{abstract}
Resumo
Objetivou-se analisar percepção sobre presença de sintomas depressivos em cuidadores familiares de idosos com doença de Alzheimer ou outras demências. Estudo exploratório-descritivo, quantitativo. Foi utilizado questionário semiestruturado e três escalas psicométricas para avaliação da sintomatologia depressiva. Amostra constituída por 51 cuidadores familiares. Análise estatística pelo teste exato de Fisher e teste para Comparação das Proporções. 50 cuidadores apresentaram sintomatologia depressiva com intensidade predominante de leve a moderada, 27 (52,9\%) percebiam-se deprimidos, $23(45,1 \%)$ não se percebiam adoecidos, embora tenham identificados possíveis fatores para sofrimento psíquico e terem relacionado o cuidado ao idoso demenciado como principal fator para seu adoecimento; $96 \%$ referiram o cuidar do idoso como causa de adoecimento. Maioria dos cuidadores apontou falta de suporte familiar, sintomas e evolução da doença como principais fatores para depressão, não receberam nenhum tipo de intervenção para seu sofrimento psíquico. Necessário diagnóstico precoce e intervenções para prevenção ao sofrimento dos cuidadores familiares.
\end{abstract}

Palavras-chave: Depressão; Cuidadores; Idosos; Demência.

\begin{abstract}
The objective was to analyze the perception of the presence of depressive symptoms in family caregivers of elderly people with Alzheimer's disease or other dementias. Exploratory-descriptive, quantitative study. A semi-structured questionnaire and three psychometric scales were used to assess depressive symptoms. Sample consisting of 51 family caregivers. Statistical analysis using Fisher's exact test and test for comparison of proportions. 50 caregivers presented depressive symptoms with predominant mild to moderate intensity, 27 (52.9\%) felt depressed, 23 (45.1\%) did not feel sick, although they identified possible factors for psychological distress and related care the demented elderly person as the main factor for their illness; $96 \%$ reported caring for the elderly as a cause of illness. Most caregivers pointed to lack of family support, symptoms and progression of the disease as the main factors for depression, they did not receive any type of intervention for their psychological suffering. Early diagnosis and interventions to prevent the suffering of family caregivers are needed.
\end{abstract}

Keywords: Depression; Caregivers; Aged; Dementia.

\section{Resumen}

El objetivo fue analizar la percepción de la presencia de síntomas depresivos en cuidadores familiares de personas mayores con enfermedad de Alzheimer u otras demencias. Estudio exploratorio-descriptivo, cuantitativo. Se utilizó un cuestionario semiestructurado y tres escalas psicométricas para evaluar los síntomas depresivos. Muestra compuesta por 51 cuidadores familiares. Análisis estadístico utilizando la prueba exacta de Fisher y la prueba de comparación de proporciones. 50 cuidadores presentaban síntomas depresivos con predominio de intensidad leve a moderada, 27 $(52,9 \%)$ se sentían deprimidos, $23(45,1 \%)$ no se sentían enfermos, aunque identificaron posibles factores de angustia psicológica y cuidados relacionados al anciano demente como principal factor de su enfermedad; El $96 \%$ informó que el cuidado de los ancianos es una causa de enfermedad. La mayoría de los cuidadores señalaron la falta de apoyo familiar, los síntomas y la progresión de la enfermedad como los principales factores de depresión, no recibieron ningún 
tipo de intervención por su sufrimiento psicológico. Se necesitan diagnósticos e intervenciones precoces para prevenir el sufrimiento de los cuidadores familiares.

Palabras clave: Depresión; Cuidadores; Anciano; Demencia.

\section{Introdução}

O envelhecimento populacional e a demência, como aspecto intrínseco a ele, configuram fenômeno global, alvos de importantes debates e pesquisas nacionais e mundiais (Mira \& Carnin, 2017; Santos, Bessa, \& Xavier, 2020; Malta, Araújo, Brito, \& Pinho, 2020).

Estima-se que a proporção de pessoas com mais de 60 anos irá dobrar até 2050, se comparado a 2015, passando de $12 \%$ para 22\%, em números absolutos passará de 900 milhões para 2 bilhões de acordo com a Organização Mundial da Saúde (Organização Pan-Americana de Saúde, 2018).

No Brasil, a população idosa cresce em velocidade maior que a mundial. É projetado que em 2050 sejam 70 milhões, crescimento de 186,9\% frente aos 24,4 milhões em 2015 (Dadalto \& Cavalcante, 2021).

Verifica-se a ocorrência de uma transição demográfica com a queda da natalidade e idade média da população passando de 23,41 anos em 1950 para 51,10 anos em 2100. Desta forma, o perfil populacional será marcado por pessoas envelhecidas, uma situação que produzirá impactos sociais e econômicos (Myrrha, Turra, \& Wajnman, 2017).

Com o processo de envelhecimento populacional, há o aumento de patologias de cunhos nutricionais, infeciosas e crônicas. Entre as doenças crônicas está a demência, uma doença caracterizada por diminuição das funções cognitivas intelectuais (perdas de memória, da abstração do raciocínio, do senso crítico e da linguagem) (Silveira \& Silva, 2020).

Dados mundiais de 2020 apontam que mais de 50 milhões de pessoas vivem com demência. Há a incidência anual de 9,9 milhões de casos, que significa um novo caso a cada 3,2 segundos (Alzheimer's Disease International, 2021). No Brasil, cerca de 1,2 milhão de pessoas vivem com demências, e a estimativa de aumento é considerável, pois o envelhecimento é o principal fator de risco para o desenvolvimento desta doença (Dadalto \& Cavalcante, 2021).

Diante deste contexto, é consensual que esta problemática traga à luz outro aspecto evidente no cenário nacional, com pouca ação efetiva por parte das políticas públicas e das ações em saúde, que é o aumento pela demanda de cuidados a idosos portadores de Doença de Alzheimer (DA) e outras demências, consequentemente fazendo com que familiares sejam cada vez mais solicitados para o cuidar.

A necessidade de cuidados ininterruptos, difícil manejo das manifestações clínicas, psiquiátricas e comportamentais, somadas às vivências dos laços emocionais, tanto positivos como negativos experienciados pelo convívio anterior a instalação da doença, produzem desgastes físico, mental, emocional e social, geradores de sobrecarga no cuidador (Luzardo, Gorini, \& Silva, 2006).

A depressão vem sendo considerada principal causa de incapacitação no mundo, com mais de 300 milhões de pessoas convivendo com este quadro, a corresponder aumento de mais de $18 \%$ entre 2005 e 2015 . A prevalência de depressão no mundo é de 4,4\%, no Brasil, considerado país com maior número de casos de depressão da América Latina, a prevalência é de 5,8\%, correspondem a 11,5 milhões da população afetada (Gonçalves et al., 2018; Organização Pan-Americana de Saúde, 2018).

Verifica-se que os cuidadores familiares são colocados à parte quanto ao planejamento das ações em saúde, pois em muitos casos o cuidador se sente desamparado e sem suporte familiar, social e pelo sistema de saúde em si, adoece e o sistema de saúde ainda não o atende como parte integrante deste idoso, que também requer e necessita de cuidados, pois não há como cuidar de um sem cuidar do outro.

Este estudo torna-se relevante porque cria espaço para reflexões e conhecimento acerca da saúde mental dos cuidadores familiares de idosos com DA ou outra demência que, no Brasil, ainda não foi desvelado pelos profissionais e organizações de 
saúde.

Este estudo teve por objetivos analisar a presença e intensidade dos sintomas depressivos em cuidadores familiares de idosos com Alzheimer ou outra demência, conhecer fatores que contribuem para surgimento dos mesmos, analisar a percepção sobre os sintomas depressivos apresentados e verificar se recebem algum tipo de intervenção.

\section{Metodologia}

Estudo descritivo-transversal, quantitativo (Marconi \& Lakatos, 2021). Pesquisa desenvolvida com cuidadores familiares participantes dos grupos de uma associação nacional de Alzheimer - seção São Paulo, localizados na zona sul do município de São Paulo/SP nos Grupos Brooklin; Moema; Paraíso e Santa Cruz. As entrevistas foram realizadas nesses locais.

Amostra não probabilística e número amostral foi definido por conveniência, constituído de no mínimo $20 \%$ do total de cuidadores familiares que frequentava os quatro grupos escolhidos no período anterior à coleta de dados. Como critério de inclusão serem familiares de indivíduo portador de demência de Alzheimer ou outra demência, que realizavam cuidados sem ser remunerado, há no mínimo 3 meses e 4 horas/dia. Obtivemos 51 cuidadores familiares que participaram do estudo.

Foram utilizados quatro instrumentos para a coleta de dados, sendo que a pesquisadora recebeu treinamento específico para aplicação das escalas psicométricas. Por meio de entrevista, foi aplicado questionário semiestruturado elaborado pelos pesquisadores, contendo informações sócio demográficas, questões relacionadas ao cuidador, aos cuidados prestados pelo mesmo e a sintomatologia depressiva.

As três escalas psicométricas usadas para coleta de dados sobre sintomatologia depressiva foram: Inventário de Depressão de Beck (IDB), Escala de Avaliação para Depressão de Hamilton (HAM-D) e Escala de Avaliação para Depressão de Montgomery- Asberg (MADRS), traduzidas e validadas no Brasil.

Os resultados das escalas psicométricas foram avaliados de acordo com escores definidos para cada escala, posteriormente submetidos à análise estatística e correlacionados com os dados sociodemográficos utilizando-se o software Minitab versão 17. Foram aplicados o teste exato de Fisher e teste de comparação das proporções.

Foi garantido cumprimento das questões éticas em pesquisa em acordo com Resolução no 466/2012 do Conselho Nacional de Saúde. A coleta de dados ocorreu no período entre novembro de 2014 a maio de 2015, após autorização da instituição onde ocorreu a coleta de dados e aprovação do projeto de pesquisa pelo Comitê de Ética em Pesquisa sob nº 528.137/ 2014.

\section{Resultados}

As características predominantes dos 51 cuidadores participantes dessa pesquisa são descritas na Tabela 1.

Constatou-se que 90,0\% dos cuidadores receberam algum tipo de ajuda para o cuidado ao idoso, tendo como principais fontes de suporte o cuidador profissional (47,0\%) ou outros familiares (31,0\%). A maioria dos cuidadores 39 (76,5\%) disse ter abdicado de algo para cuidar de seu familiar, como liberdade, lazer, trabalho entre outros; 92\% referiu possuir boas condições de moradia e $70 \%$ referiu possuir boas condições econômicas, fato que pode justificar que quase metade dos cuidadores possuíam suporte de profissional. No grau de parentesco, dos 40 que informaram ser filhos, apenas três do sexo masculino. 
Tabela 1. Características sociodemográficas de cuidadores familiares de idosos com Alzheimer ou outra demência. São Paulo (SP), Brasil, 2021.

\begin{tabular}{|c|c|c|}
\hline Variáveis predominantes & $\mathbf{n}$ & $\%$ \\
\hline \multicolumn{3}{|l|}{ Gênero } \\
\hline Feminino & 45 & 88,0 \\
\hline \multicolumn{3}{|l|}{ Faixa etária } \\
\hline$>50$ & 41 & 80,0 \\
\hline \multicolumn{3}{|l|}{ Estado civil } \\
\hline Casada (o) & 25 & 49,0 \\
\hline Solteira (o) & 11 & 21,0 \\
\hline \multicolumn{3}{|l|}{ Grau de Escolaridade } \\
\hline Superior Completo & 39 & 76,0 \\
\hline \multicolumn{3}{|l|}{ Religião } \\
\hline Católica & 25 & 49,0 \\
\hline \multicolumn{3}{|l|}{ Grau de parentesco } \\
\hline Filha (o) & 40 & 78,0 \\
\hline \multicolumn{3}{|l|}{ Situação Ocupacional } \\
\hline Aposentado & 18 & 35,0 \\
\hline Empregado & 15 & 29,0 \\
\hline \multicolumn{3}{|l|}{ Fonte de renda } \\
\hline Trabalho próprio & 24 & 47,0 \\
\hline Aposentadoria & 18 & 35,0 \\
\hline \multicolumn{3}{|l|}{ Com quem reside } \\
\hline Reside com idoso & 35 & 68,0 \\
\hline \multicolumn{3}{|c|}{ Tempo como cuidador (meses) } \\
\hline$>60$ & 22 & 43,0 \\
\hline 13 a 24 & 13 & 25,0 \\
\hline \multicolumn{3}{|l|}{ Carga horária como cuidador } \\
\hline 4 a 5 horas/dia & 21 & 41,0 \\
\hline$>14$ horas/dia & 19 & 37,0 \\
\hline \multicolumn{3}{|l|}{ Quantos dias por semana } \\
\hline 7 & 31 & 60,0 \\
\hline
\end{tabular}

Fonte: Elaborada pelos autores com dados da pesquisa.

A Tabela 2 apresenta resultados detectados pelas escalas psicométricas para entrevistados com e sem diagnóstico prévio para depressão. A maioria dos cuidadores no IDB apresentaram escores negativos, porém quando responderam outras escalas e questões relacionadas a sintomatologia depressiva relatada e questionados se estar deprimido estava relacionado ao fato de ter que cuidar de seu familiar, 49 (96,0\%) afirmaram que sim, divergindo dos resultados obtidos pelo IDB. 
Tabela 2. Resultados para presença e intensidade de sintomatologia depressiva detectada pelas escalas psicométricas. São Paulo (SP), Brasil, 2021.

\begin{tabular}{lrr}
\hline & Com Diagnóstico prévio & Sem Diagnóstico prévio \\
\hline Escores das escalas psicométricas & $16(31,4 \%)$ & $35(68,6 \%)$ \\
\hline IDB & & \\
\hline Sem Depressão & $4(7,8 \%)$ & $29(56,8 \%)$ \\
Depressão Leve & $9(17,7 \%)$ & $3(5,9 \%)$ \\
Depressão Moderada & $3(5,9 \%)$ & $3(5,9 \%)$ \\
Depressão Grave & - & - \\
\hline HAM-D & - & $1(2,0 \%)$ \\
Sem Depressão & $3(5,9 \%)$ & $17(33,3 \%)$ \\
Depressão Leve & $12(23,5 \%)$ & $11(21,5 \%)$ \\
Depressão Moderada & $1(2,0 \%)$ & $6(11,8 \%)$ \\
Depressão Grave & - & $1(2,0 \%)$ \\
\hline MADRS & - & $19(37,2 \%)$ \\
\hline Sem Depressão & $3(5,9 \%)$ & $12(23,5 \%)$ \\
Depressão Leve & $13(25,5 \%)$ & $3(5,9 \%)$ \\
Depressão Moderada & - & \\
Depressão Grave & & \\
\hline
\end{tabular}

Fonte: Elaborada pelos autores com dados da pesquisa.

Dos 51 cuidadores, obtiveram-se 83 respostas aos fatores considerados determinantes para o adoecimento psíquico, conforme Tabela 3.

Tabela 3. Fatores determinantes para surgimento de sintomas depressivos. São Paulo (SP), Brasil, 2021.

\begin{tabular}{lcr}
\hline Fatores & n & \% \\
\hline Falta de suporte familiar & 25 & 30,1 \\
Sintomas/Evolução da demência & 24 & 29,0 \\
Falta de dinheiro/infraestrutura & 15 & 18,1 \\
Perda/privação da liberdade & 10 & 12,0 \\
Outros fatores & 9 & 10,8 \\
\hline Total & 83 & 100,0 \\
\hline
\end{tabular}

Fonte: Elaborada pelos autores com dados da pesquisa.

Relatados em "Outros fatores": "rotina de cuidados” (4), "preocupação" (2) e "falta de preparo para cuidar" (3).

Para comparar os grupos com ou sem diagnóstico prévio foram utilizados o teste de Fisher para a intensidade da depressão entre as escalas psicométricas, a comparar se percentuais nesses grupos seriam inferenciados como iguais. Pode-se dizer que houve associação entre instrumentos de avalição do observador (HAM-D e MADRS) visto que p-value $\leq 0,05$.

Observa-se diferença nos percentuais para quem possuía e não possuía depressão prévia, pelo IDB, HAM-D e MADRS. Os percentuais de com ou sem depressão prévia variam de acordo com o grau da escala (intensidade dos sintomas), conforme Tabela 4 . 
Tabela 4. Resultados comparativos para depressão prévia e intensidade de depressão entre escalas IDB, HAM-D e MADRS. São Paulo (SP), Brasil, 2021

\begin{tabular}{|c|c|c|c|c|c|}
\hline & \multirow{2}{*}{ Grupo } & \multicolumn{2}{|c|}{ Depressão Prévia } & \multirow{2}{*}{$p$-value $*$} & \multirow{2}{*}{$p$-value $e^{* *}$} \\
\hline & & Não $(n=35)$ & $\operatorname{Sim}(n=16)$ & & \\
\hline \multirow{4}{*}{ IDB } & Sem Depressão & 29 & 4 & 0,001 & 0,001 \\
\hline & Depressão Leve & 3 & 9 & & \\
\hline & Depressão Moderada & 3 & 3 & & \\
\hline & Depressão Grave & - & - & & \\
\hline \multirow{4}{*}{ HAM } & Sem Depressão & 1 & - & $\mathbf{0 , 0 3 6}$ & 0,036 \\
\hline & Depressão Leve & 17 & 3 & & \\
\hline & Depressão Moderada & 11 & 12 & & \\
\hline & Depressão Grave & 6 & 1 & & \\
\hline \multirow{4}{*}{ MADRS } & Sem Depressão & 1 & - & 0,019 & 0,019 \\
\hline & Depressão Leve & 19 & 3 & & \\
\hline & Depressão Moderada & 12 & 13 & & \\
\hline & Depressão Grave & 3 & - & & \\
\hline
\end{tabular}

* Teste para igualdade das proporções Sem Depressão Prévia.

** Teste para igualdade das proporções Com Depressão Prévia.

Fonte: Elaborada pelos autores com dados da pesquisa.

Utilizado teste de Fisher para medidas atributo para comparar percentuais de depressão na HAM-D e MADRS. Houve diferença nos percentuais de depressão grave na escala HAM-D nos atributos grau de escolaridade (p -value=0,042) e principal fonte de renda $(\mathrm{p}$-value $=0,035$ ). Houve diferença nos percentuais de sem depressão no atributo tempo como cuidador/meses $(\mathrm{p}$ value $=0,001)$.

$\mathrm{Na}$ escala MADRS verificou-se diferença nos percentuais de depressão leve para atributo faixa etária $(0,045)$ e percentuais de sem depressão no atributo tempo como cuidador/meses $(0,001)$.

Somente $13(25,49 \%)$ afirmaram receber intervenção para sintomas depressivos relatados, sendo psicoterapia realizada por psicólogos a intervenção mais citada. Dos 13 respondentes, 9 sentiram necessidade de suporte psicológico e buscaram ajuda por conta própria, 2 cuidadores procuraram apoio psicológico devido incentivo de amigos, 2 nada relataram.

As principais justificativas para nenhuma intervenção foram: "não ter tempo para cuidar da saúde"; "não sentir necessidade de procurar ajuda"; "acha normal ter sintomas depressivos e que com o tempo vai melhorar"; "reconhece que precisa de ajuda, mas posterga por medo/vergonha do diagnóstico"; "falta de coragem para pedir ajuda", "falta de dinheiro para pagar um tratamento".

Dos $50(98,0 \%)$ que apresentaram resultado para sintomatologia depressiva, 27(52,9\%) se percebiam e $23(45,1 \%)$ não se percebiam deprimidos.

Comparando-se os dados anteriores aos do IDB, verifica-se que maioria dos cuidadores não admitiram ou não acreditavam possuir sintomas depressivos, apesar de citarem diversos fatores relacionados ao cuidado como determinantes para seu adoecimento psíquico.

\section{Discussão}

Os resultados evidenciam alto índice de cuidadores com sintomas depressivos de leve a moderado, detectados pelas escalas de HAM-D e MADRS, muito acima do encontrado na literatura (Santos-Orlandi et al., 2019; Terassi, Rossetti, Luchesi, Gramani-Say, Hortense, \& Pavarini, 2020). Em nosso estudo, 31,3\% dos entrevistados relataram diagnóstico prévio, e dos que referiram não possuir, apenas um não apresentou resultados para sintomatologia depressiva na HAM e MADRS. 
Os instrumentos HAM-D e MADRS mostraram resultados congruentes e também quando associados aos dados do questionário, que confirmaram os entrevistados estarem adoecidos e assim não se perceberem, à semelhança do encontrado em outras pesquisas (Fernandes \& Marcolan, 2017; Oliveira, Mazzaia, \& Marcolan, 2015).

Há similaridade com resultados obtidos por outros estudos que mostraram altos níveis de sobrecarga física e emocional em cuidadores familiares de idosos com DA o que contribuiu significativamente para presença de sintomas depressivos (Henriques, Cabana, \& Montarroyos, 2018; Souza, Ferreira, Gonçalves, Polaro, \& Fernandes, 2020).

Outro estudo objetivou caracterizar diferenças de carga de cuidador em pacientes com demência de Alzheimer e demência vascular (DVa), para melhorar o aconselhamento e plano de manejo de cuidados, revelando níveis maiores de sobrecarga em cuidadores de idosos com DA (D'Onofrio et al., 2015).

Não foram encontrados registros de pesquisas envolvendo a percepção de cuidadores informais de idosos acerca de sintomas depressivos, mas dados relatando sobrecarga referida por estes, que estavam atrelados ao sofrimento psíquico do cuidador e fortemente associada a depressão (Lino et al., 2016; Liu et al., 2017).

Cuidadores de pessoas com demência experimentaram sentimento de maior responsabilidade e sentimento mais forte de ser preso em seu papel de cuidarem em relação a outros cuidadores (Fisher et al., 2011).

Há estudos que corroboram esta pesquisa pela predominância de cuidadores do sexo feminino, filhas, com idade média de 55 anos ou mais, casadas, predomínio religião católica, coabitavam com o idoso, alguns possuíam outra ocupação além do cuidado e afazeres domésticos (Jeske, Blasi, Casarin, \& Ilha, 2020; Faria, Scardoelli, Castro, \& Nishida, 2017; Oliveira \& Moura, 2017).

No presente estudo há predominância do gênero feminino, evidenciando realidade em que a mulher, mesmo muitas vezes assumindo diversos papéis, dentro e fora do lar, acaba também por assumir papel de cuidadora principal, função que deveria ser compartilhada com demais membros familiares, e influencia para a exaustão frente a responsabilidade imposta a esta cuidadora (Silveira \& Silva, 2020).

Relativo às características sobre nível de escolaridade, condições econômicas e moradia dos cuidadores desta pesquisa, foram encontradas discrepâncias a outros estudos que mostram cuidadores com baixo nível de escolaridade e situação econômica e de moradia complexas (Lino et al., 2016; Alves et al., 2015; Fauth et al., 2012).

Quanto ao tempo em meses, quantidade de dias por semana e carga horária diária dedicadas aos familiares, os dados vão ao encontro da literatura, pois revelam que os cuidadores familiares dedicam boa parte de seu tempo e de suas vidas na prestação de cuidados, ficando em média mais de 8 horas por dia, no mínimo 5 dias por semana e há mais de 5 anos (Alves et al., 2015).

Estudo transversal na China com 309 cuidadores informais familiares revelou que a maioria deles exercia cuidado em tempo integral, sem auxílio, possuindo pouco tempo para cuidar de si, fato associado a níveis altos de ansiedade e depressão e a uma vida insatisfatória (Liu et al., 2017).

Em estudo comparativo, realizado com cuidadores familiares informais primários e secundários que frequentavam associação similar de Maringá-PR, amostra com 40 cuidadores, constatou que cuidadores primários exerciam esta função em média por sete anos, apresentando maior sobrecarga se comparado ao grupo de cuidadores secundários (Borghi, Castro, Marcon, $\&$ Carreira, 2013).

A maioria dos cuidadores de nosso estudo revelou ter abdicado de algo para exercer o cuidado, mesmo aqueles com condições de ter alguém para ajudá-lo, sentiam muitas vezes dificuldades em ter momentos de lazer, quando os tinham, sentiamse culpados por estar a fazer algo considerado errado, ao deixar o familiar aos cuidados de outrem, à semelhança do encontrado na literatura (Oliveira \& Moura, 2017).

Constata-se alto índice de cuidadores familiares que recebiam ajuda de profissionais ou de familiares, o que é incomum, 
quando comparado a outra pesquisa, em que a maioria dos cuidadores afirmou não receber ajuda para o cuidado, sendo eles muitas vezes os únicos responsáveis pelo cuidado e por todas as outras tarefas cotidianas (Liu et al., 2017).

A maioria das famílias brasileiras não possui condições financeiras para manter o cuidador formal, visto ser bem oneroso, principalmente, após a legalização da profissão, inviabilizando a contratação de tais profissionais por famílias de baixa renda, predominante em nossa sociedade (Silveira \& Silva, 2020; Diniz et al., 2018; Lima et al., 2021).

Os cuidadores que prestam cuidados por tempo prolongado e, principalmente, aqueles que não possuem ajuda, estão mais expostos aos riscos de adoecimento, pois, se encontram mais sobrecarregados, principalmente no caso das mulheres, que acumulam diversos papéis, além da função de cuidadora (Gonçalves, Alvarez, Sena, Santana, \& Vicente, 2006).

Ressalta-se que sintomas depressivos no cuidador podem passar despercebidos no início da demência, devendo-se observar com cautela o cuidador, desde o diagnóstico de seu familiar como durante todo percurso da demência (Välimäki, Vehviläinen-Julkunen, Pietilä, \& Pirttilä, 2009).

A identificação precoce e intervenções a esta população podem reduzir seus níveis de depressão e morbidade psicológica, como constataram pesquisadores quanto a incidência de depressão poderia ser reduzida em 72,3\%, se os grupos de risco (cuidadores familiares) fossem alvo de programas de intervenção efetiva (Joling et al., 2012).

Ressalte-se importância das equipes de saúde ao lidar com esta população, pois, muitas vezes, ela só possui o suporte destas que precisam estar atentos para todas dificuldades relatadas pelo cuidador, ser capaz de intervir naquelas que passam despercebidas (Faria, Scardoelli, Castro, \& Nishida, 2017).

Compreender o sofrimento do cuidador, realizar diagnóstico e intervenções precoces é fundamental para aliviar e prevenir agravos à saúde, pois apenas compreensão não alivia o sofrimento de quem não terá perspectiva de futuro, com o peso da desesperança de cura de seu familiar, sentindo-se abandonado à própria sorte, como muitos dizem "cumprindo a vontade de Deus".

O trabalho em conjunto das equipes de saúde, redes de apoio à saúde mental e grupos de ajuda mútua constitui importante rede de atuação e suporte a esta população, agregam valor significativo à vida destas pessoas, carentes de suporte humano e afetivo.

Dados das escalas psicométricas vão ao encontro da literatura ao mostrar congruência ao serem analisados entre si, permitindo maior confiabilidade dos mesmos. Detectamos que os cuidadores, por diversos motivos, referiram não se perceber, ou mesmo não se aceitar com sintomas depressivos, mesmo relatando que cuidar de familiar com DA ou outras demências contribui para surgimento ou agravamento de sintomas depressivos; citaram diversos fatores contribuintes encontrados na literatura (Oliveira, Mazzaia, \& Marcolan, 2015; Fernandes \& Marcolan, 2017).

A dificuldade encontrada por essa população, em se perceber e se assumir com depressão, revela o estigma que abarca o transtorno ainda hoje. Os próprios indivíduos portadores de transtornos depressivos sentem-se estigmatizados e por vezes envergonhados (Gonçales \& Machado, 2007).

O desgaste físico e mental foi significativamente correlacionado com os sintomas neuropsiquiátricos do Alzheimer e como principais fatores preditivos de ansiedade e depressão à semelhança de outros achados (Lou et al., 2015).

Os problemas de saúde do cuidador configuram-se pelo somatório de fatores relacionados à tensão emocional, esforço físico constante e suas outras responsabilidades (Silveira \& Silva, 2020).

Pesquisas que se dedicam a verificar fatores físicos e psicológicos que afetam cuidadores de idosos com Alzheimer apontam a falta de apoio por parte da família, o prognóstico de não melhora do quadro de seu familiar e o reconhecimento sobre a necessidade de capacitação para este cuidado. A qualidade de vida dos cuidadores é afetada em aspectos físico e emocional, saúde mental, dor física e aspecto social (Caldeira \& Ribeiro, 2004; Pinto, Barbosa, Ferreti, Souza, Fram, \& Belasco, 2009).

A difícil tarefa de cuidar de idosos com quadro demencial faz com que apresentar sintomas depressivos e alterações 
cognitivas, em geral, se torne constante nesta população. O sofrimento do cuidador tem seu término com o óbito de uma das partes, sendo está triste realidade (Bianchi, Flesch, Alves, Batistoni, \& Neri, 2016).

Vários estudos envolvendo cuidadores apresentaram resultados semelhantes aos nossos, e evidenciaram a importância de se ofertar apoio a este grupo, rever as estratégias de intervenções e tratamento com intuito de reduzir a ansiedade e depressão nesta população (Mendes, Faustino, Santos, \& Cruz, 2020; Martins, Corrêa, Caparrol, Santos, Brugnera, \& Gratão, 2019; Silva et al., 2020).

Como profissionais da saúde, devemos estar atentos às estratégias de enfrentamento utilizadas pelo cuidador e intervir de forma a oferecer ou complementar este suporte, por meio de encaminhamento a profissionais especializados e outras redes de apoio, trabalhar na identificação e solução das necessidades a serem sanadas. A literatura aponta diversos tipos de intervenções para cuidadores de idosos com doenças neurodegenerativas sendo mais comum: grupos de apoio, intervenções psicoeducacionais, terapia familiar e individual (Cruz \& Hamdan, 2008).

Pesquisa comparou dois programas de intervenção psicoeducativa, com 168 cuidadores informais, com resultados positivos para diminuição de distresse psicológico, ressalta importância da implementação em rede deste tipo de programa a este núcleo (Alves, Brandão, Teixeira, Azevedo, Duarte, Ribeiro, \& Paúl, 2015).

Verificou-se que o cuidador não se percebendo ou não querendo assumir o quadro depressivo, demonstra por meio de palavras e atitudes que necessita de suporte, e, de diferentes formas, pede socorro. Intervir previamente nesses casos por meio de avaliação do cuidador por parte do profissional que cuida do indivíduo com DA e outras demências, é medida premente a ser padronizada.

$\mathrm{Na}$ Enfermagem o ensino é centrado com ênfase nas técnicas, que privilegiam as habilidades manuais e de gestão em relação às interpessoais, o objeto deixa de ser o cuidado centrado no paciente e família para a tarefa, a refletir na assistência prestada (Pires, 2005).

Como limitações do estudo, considerou-se o tamanho da amostra que não permite amplas generalizações, a classe social dos cuidadores como possível viés de seleção, pois permitiu a participação de pessoas com níveis altos de escolaridade e boas condições econômicas, mas deixou de fora cuidadores que devido as condições de maior vulnerabilidade não conseguiam frequentar os grupos.

\section{Considerações Finais}

Os resultados do estudo fornecem subsídios para direcionar ações em saúde, sobretudo dos enfermeiros, para revelar estes pacientes "ocultos" e atendimento as necessidades. Profissionais de enfermagem da atenção primária têm relevância para ajudar idosos com demência e cuidadores, identificando e atuando precocemente no sofrimento.

Identificou-se pelas escalas psicométricas, que à exceção de um, os cuidadores familiares apresentaram sintomatologia depressiva, de intensidade leve a grave, resultados superiores aos da população geral.

Aspectos relacionados aos sintomas e evolução da demência e à falta de suporte familiar foram os mais apontados pela maioria dos cuidadores para seu adoecimento.

Verificou-se considerável parte dos cuidadores familiares sem se perceberem adoecidos, embora tenham identificados fatores contribuintes ao sofrimento psíquico apresentado e terem o cuidado ao idoso como principal fator para seu adoecimento.

A maioria não recebia intervenções para a sintomatologia apresentada e referiram falta de tempo, coragem e dinheiro para se tratar; por vergonha e medo de receber diagnóstico de depressão; por acreditarem ser normal sentir tais sintomas diante da circunstância vivenciada.

Necessitamos de estratégias em saúde para assegurar assistência eficaz à saúde desse binômio, à família e seu contexto, 
frente ao envelhecimento desenfreado que ocorre na população.

\section{Fomento}

Pesquisa realizada por meio de concessão de bolsa de mestrado pelo Conselho Nacional de Desenvolvimento Científico e Tecnológico - CNPq.

\section{Referências}

Alves, S., Brandão, D., Teixeira, L., Azevedo, M. J., Duarte, M., Ribeiro, Ó., \& Paúl, C. (2015). Intervenções psicoeducativas e distresse picológico em cuidadores informais: Análise de dois projetos comunitários. Revista E-Psi, 5(1), 94-112.

Alzheimer's Disease International. Dementia statistics. Recuperado de: https://www.alzint.org/about/dementia-facts-figures/dementia-statistics/ , em 19 mar. 2021.

Bianchi, M., Flesch, L. D., Alves, E. V. C., Batistoni, S. S. T., \& Neri, A. L. (2016). Indicadores psicométricos da Zarit Burden Interview aplicada a idosos cuidadores de outros idosos. Revista Latino-Americana de Enfermagem, 24, e2835. https://doi.org/10.1590/1518-8345.1379.2835

Borghi, A. C., Castro, V. C., Marcon, S. S., \& Carreira, L. (2013). Sobrecarga de familiares cuidadores de idosos com doença de Alzheimer: um estudo comparativo. Revista Latino-Americana de Enfermagem, 21(4), 1-7.

Caldeira, A. P.S., \& Ribeiro, R. C. H. M. (2004). O enfrentamento do cuidador do idoso com Alzheimer. Arquivo Ciências da Saúde, 11 (2), 1-5.

Cruz, M. N., \& Hamdan, A. C. (2008). O impacto da doença de Alzheimer no cuidador. Psicologia em Estudo, 13(2), 223-229. https://dx.doi.org/10.1590/S141373722008000200004

D'Onofrio, G., Sancarlo, D., Addante, F., Ciccone, F., Cascavilla, L., Paris, F., ... \& Pilotto, A. (2015). Caregiver burden characterization in patients with Alzheimer's disease or vascular dementia. International journal of geriatric psychiatry, 30(9), 891-899. https://doi.org/10.1002/gps.4232

Dadalto, E. V., \& Cavalcante, F. G. C. (2021). O lugar do cuidador familiar de idosos com doença de Alzheimer: uma revisão de literatura no Brasil e Estados Unidos. Ciência \& Saúde Coletiva, 26(1), 147-157. https://dx.doi.org/10.1590/1413-81232020261.38482020

Diniz, M. A. A., Melo, B. R. S., Neri, K. H., Casemiro, F. G., Figueiredo, L. C., Gaioli, C. C. L. O., \& Gratão, A. C. M. (2018). Estudo comparativo entre cuidadores formais e informais de idosos. Ciência \& Saúde Coletiva, 23(11), 3789-3798. https://doi.org/10.1590/1413-812320182311.16932016

Faria, E. B. A., Scardoelli, M. G., Castro, V. C. de, \& Nishida, F. S. (2017). Vivências de Cuidadores Familiares de Pessoas Idosas com Doença de Alzheimer. Ciência, Cuidado E Saúde, 16(1). https://doi.org/10.4025/cienccuidsaude.v16i1.31004

Fauth, E., Hess, K., Piercy, K., Norton, M., Corcoran, C., Rabins, P., ... \& Tschanz, J. (2012). Caregivers' relationship closeness with the person with dementia predicts both positive and negative outcomes for caregivers' physical health and psychological well-being. Aging \& mental health, 16(6), 699-711. https://doi.org/10.1080/13607863.2012.678482

Fernandes, D. M., \& Marcolan, J. F. (2017). Trabalho e sintomatologia depressiva em enfermeiros da Estratégia de Saúde da Família. SMAD Revista Eletrônica Saúde Mental Álcool E Drogas, 13(1), 37-44. https://doi.org/10.11606/issn.1806-6976.v13i1p37-44

Fisher, G. G., Franks, M. M., Plassman, B. L., Brown, S. L., Potter, G. G., Llewellyn, D., ... \& Langa, K. M. (2011). Caring for individuals with dementia and cognitive impairment, not dementia: findings from the aging, demographics, and memory study. Journal of the American Geriatrics Society, 59(3), 488-494. https://doi.org/10.1111/j.1532-5415.2010.03304.x

Gonçalves, A. M. C., Teixeira, M. T. B., Gama, J. R. A., Lopes, C. S., Silva, G. A., Gamarra, C. J., ... Machado, M. L. S. M. (2018). Prevalência de depressão e fatores associados em mulheres atendidas pela Estratégia de Saúde da Família. Jornal Brasileiro de Psiquiatria, 67(2), 101-109. https://doi.org/10.1590/00472085000000192

Gonçales, C. A. V., \& Machado, A. L. R. (2007). Depressão, o mal do século: De que século? Revista de Enfermagem da UERJ, 15 (2), $298-304$.

Gonçalves, L. H. T., Alvarez, A. M., Sena, E. L. S., Santana, L. W. S., \& Vicente, F. R. (2006). Perfil da família cuidadora de idoso doente/fragilizado do contexto sociocultural de Florianópolis, SC. Texto \& Contexto - Enfermagem, 15(4), 570-577. https://dx.doi.org/10.1590/S0104-07072006000400004

Henriques, R. T. M., Cabana, M. C. F. L., \& Montarroyos, U. R. (2018). Prevalência de Transtornos Mentais Comuns e sua associação com a sobrecarga em cuidadores familiares de idosos. Mental, 12(22), 35-52. Recuperado em 02 de abril de 2021, de http://pepsic.bvsalud.org/scielo.php?script=sci_arttext\&pid=S1679-44272018000100004\&lng=pt\&tlng=pt.

Jeske, T. G., Blasi, T. C., Casarin, F., \& Ilha, S. (2020). Compreensão do cuidado na alimentação de familiares/cuidadores de pessoas idosas com a doença de Alzheimer. Research, Society and Development, 9(7), e235973913. https://doi.org/10.33448/rsd-v9i7.3913

Joling, K. J., Smit, F., van Marwijk, H. W., van der Horst, H. E., Scheltens, P., Schulz, R., \& van Hout, H. P. (2012). Identifying target groups for the prevention of depression among caregivers of dementia patients. International psychogeriatrics, 24(2), 298-306. https://doi.org/10.1017/S1041610211001633

Lima, L. T. ., Lira, P. F. ., Oliveira, V. R. de ., Lima, L. T. ., Ribeiro , E. da C. ., Leite , J. C. de S. ., \& Silva , J. P. X. . (2021). Knowledge of the caregiver on bathing in bed in a long-term institution for the elderly. Research, Society and Development, 10(2), e54510212760. https://doi.org/10.33448/rsd-v10i2.12760 
Lino, V. T. S., Rodrigues, N. C. P., Camacho, L. A. B., O’Dwyer, G., Lima, I. S., Andrade, M. K. N., \& Atie, S. (2016). Prevalência de sobrecarga e respectivos fatores associados em cuidadores de idosos dependentes, em uma região pobre do Rio de Janeiro, Brasil. Cadernos de Saúde Pública, 32 (6), e00060115. https://doi.org/10.1590/0102-311X00060115

Liu, S., Li, C., Shi, Z., Wang, X., Zhou, Y., Liu, S., ... \& Ji, Y. (2017). Caregiver burden and prevalence of depression, anxiety and sleep disturbances in Alzheimer's disease caregivers in China. Journal of clinical nursing, 26(9-10), 1291-1300. https://doi.org/10.1111/jocn.13601

Lou, Q., Liu, S., Huo, Y. R., Liu, M., Liu, S., \& Ji, Y. (2015). Comprehensive analysis of patient and caregiver predictors for caregiver burden, anxiety and depression in Alzheimer's disease. Journal of clinical nursing, 24(17-18), 2668-2678. https://doi.org/10.1111/jocn.12870

Luzardo, A. R., Gorini, M. I. P. C., \& Silva, A. P. S. S. (2006). Características de idosos com doença de Alzheimer e seus cuidadores: uma série de casos em um serviço de neurogeriatria. Texto \& Contexto - Enfermagem, 15(4), 587-594. https://doi.org/10.1590/S0104-07072006000400006

Malta, E. M. B. R., Araújo, D. D., Brito, M. F. S. F., \& Pinho, L. (2020). Práticas de profissionais da Atenção Primária à Saúde (APS) no cuidado a idosos com demência. Interface - Comunicação, Saúde, Educação, 24(1), e190449. https://doi.org/10.1590/Interface.190449.

Marconi, M.A., \& Lakatos, E.M.. (2021). Fundamentos da metodologia científica. (9a ed), Atlas.

Martins, G., Corrêa, L., Caparrol, A. J. S., Santos, P. T. A., Brugnera, L. M., \& Gratão, A. C. M. (2019). Características sociodemográficas e de saúde de cuidadores formais e informais de idosos com Doença de Alzheimer. Escola Anna Nery, 23(2), e20180327. https://doi.org/10.1590/2177-9465-ean-2018-0327

Mendes, G. A., Faustino, A. M., Santos, X. T. B., \& Cruz, K. C. T. (2020). Apoio a cuidadores familiares de idosos com demência. Brazilian Journal of Development, 6(10), 76828-76839. https://doi.org/10.34117/bjdv6n10-201

Mira, C., \& Carnin, A. (2017). Histórias sobre o convívio com a Doença de Alzheimer: contribuições da noção de referenciação para a análise de narrativas no contexto de interações de um Grupo de Apoio. Cadernos De Estudos Linguísticos, 59(1), 157-174. https://doi.org/10.20396/cel.v59i1.8648426.

Myrrha, L. J. D., Turra, C. M., Wajnman, S. (2017). A contribuição dos nascimentos e óbitos para o envelhecimento populacional no Brasil, 1950 a 2100. Revista Latinoamericana de Población, 11(20), 37-54.

Oliveira, F. P., Mazzaia, M. C., \& Marcolan, J. F. (2015). Sintomas de depressão e fatores intervenientes entre enfermeiros de serviço hospitalar de emergência. Acta Paulista de Enfermagem, 28(3), 209-215. https://dx.doi.org/10.1590/1982-0194201500036

Oliveira, S. C. C., \& Moura, P. R. (2017). Análise reflexiva sobre a capacidade de autocuidado dos cuidadores domiciliares. Revista da Faculdade de Ciências Médicas de Sorocaba, 19(1), 15-18.

Organização Pan-Americana de Saúde. (2018). Folha informativa - Depressão. Recuperado de: https://www.paho.org/bra/index.php?option=com_content\&view=article\&id=5635:folha-informativa-depressao\&Itemid=1095, em 08 abr. 2021.

Organização Pan-Americana de Saúde. (2018). Folha informativa - Envelhecimento e saúde. Recuperado de: https://www.paho.org/bra/index.php?option=com_content\&view=article\&id=5661:folha-informativa-envelhecimento-esaude\&Itemid=820\#: :text=Entre\%202015\%20e\%202050\%2C\%20a,de\%20baixa\%20e\%20m\%C3\%A9dia\%20renda., em 08 abr. 2021.

Pinto, M. F., Barbosa, D. A., Ferreti, C. E. L., Souza, L. F., Fram, D. S., \& Belasco, A. G. S. (2009). Qualidade de vida de cuidadores de idosos com doença de Alzheimer. Acta Paulista de Enfermagem, 22(5), 652-657. https://doi.org/10.1590/S0103-21002009000500009

Pires, M. R. G. M. (2005). Politicidade do cuidado como referência emancipatória para a enfermagem: conhecer para cuidar melhor, cuidar para confrontar, cuidar para emancipar. Revista Latino-Americana de Enfermagem, 13(5), 729-736. https://dx.doi.org/10.1590/S0104-11692005000500018

Santos, C. S., Bessa, T. A., \& Xavier, A. J. (2020). Fatores associados à demência em idosos. Ciência \& Saúde Coletiva, 25(2), 603-611. https://doi.org/10.1590/1413-81232020252.02042018.

Santos-Orlandi, A. A., Brigola, A. G., Ottaviani, A. C., Luchesi, B. M., Souza, É. N., Moura, F. G., ... \& Pavarini, S. C. I. (2019). Idosos cuidadores de idosos: fragilidade, solidão e sintomas depressivos. Revista Brasileira de Enfermagem, 72(Suppl. 2), 88-96. https://dx.doi.org/10.1590/0034-7167-2018-0137

Silva, P. D. M., Castro, J. M., Martins, R. E. C., Galinari, P. C., Azevedo, M. A., Oliveira, T. V. C., ... \& Costa, W. J. T. (2020). O impacto da doença de Alzheimer na vida do cuidador. Revista Eletrônica Acervo Enfermagem, 2, e2353. https://doi.org/10.25248/reaenf.e2353.2020

Silveira, A. G., \& Silva, D. A. (2020). Sobrecarga dos familiares no cuidado ao portador de demência senil: uma revisão integ rativa. Research, Society and Development, 9(6), e179963671. http://dx.doi.org/10.33448/rsd-v9i6.3671

Sousa, S. M. L., Ferreira, D. F., Gonçalves, L. H. T., Polaro, S. H. I., \& Fernandes, D. S. (2020). Sobrecarga do cuidador familiar da pessoa idosa com Alzheimer. Enfermagem Brasil, 19(3), 146-252. Recuperado em 02 de abril de 2021, de https://www.researchgate.net/profile/DaianeFernandes/publication/342948260_Sobrecarga_do_cuidador_familiar_da_pessoa_idosa_com_Alzheimer/links/5f73c01a92851c14bca031c1/Sobrecarga-docuidador-familiar-da-pessoa-idosa-com-Alzheimer.pdf

Terassi, M., Rossetti, E. S., Luchesi, B. M., Gramani-Say, K., Hortense, P., \& Pavarini, S. C. I.. (2020). Fatores associados aos sintomas depressivos em idosos cuidadores com dor crônica. Revista Brasileira de Enfermagem, 73(1), e20170782. https://doi.org/10.1590/0034-7167-2017-0782

Välimäki, T. H., Vehviläinen-Julkunen, K. M., Pietilä, A. M., \& Pirttilä, T. A. (2009). Caregiver depression is associated with a low sense of coherence and health-related quality of life. Aging \& mental health, 13(6), 799-807. https://doi.org/10.1080/13607860903046487 